\title{
LOS NIÑOS Y NIÑAS: ¿CIUDADANOS DE HOY O DE MAÑANA?
}

\author{
JOSEFINA FERNÀNDEZ I BARRERA \\ Departamento de Trabajo Social y Servicios Sociales. Universidad de Barcelona.
}

\section{RESUMEN}

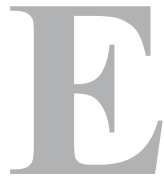

ste artículo es fruto de la tesis doctoral titulada Els infants com a ciutadans ${ }^{1}$, defendida en la Universidad de Barcelona el año 2005 para la obtención del doctorado en Sociología. La tesis defendida, respondiendo a la pregunta que encabeza este artículo, es que los niños y niñas son ciudadanos del presente a quienes debemos tener en cuenta como un colectivo importante. Es a partir de la aprobación de la Convención de las NU de los derechos del niño, el 20 de noviembre de 1989, que se reconoce a los niños y niñas como sujetos de derechos y, especialmente, el derecho de participar en todos aquellos temas que les afecten. Los objetivos del estudio parten de la convicción de que, a pesar de que el mundo de la infancia se ha ido reconociendo socialmente a partir de finales del siglo $\mathrm{XX}$, los niños y niñas aún son unos grandes desconocidos. El objetivo principal que se perseguía era identificar la participación de la infancia en la vida social, analizar cómo los niños y niñas pueden influir en la generación de nuevas formas de participación y relacionar esta participación con la noción moderna de ciudadanía. Los fundamentos metodológicos sobre los que se basó el estudio han sido básicamente de tipo etnográfico, centrándose en la observación participante de diversos espacios donde se considera que los niños y niñas tienen oportunidad de participar y en entrevistas en profundidad realizadas a personas adultas especialistas en infancia. A su vez, se contrastó con el análisis de textos. En este artículo, se procede a tratar las nociones de infancia, participación y ciudadanía y a destacar algunos de los espacios de participación de la infancia, tratados en el estudio, como son la familia, los ámbitos educativos y el tiempo libre.

Palabras clave: infancia, participación, ciudadanía. 


\begin{abstract}
This article is the result of a doctoral thesis titled Els infants com a ciutadans (Children as citizens) that was defended at the University of Barcelona in 2005 in order to obtain a $\mathrm{PhD}$ in Sociology. The thesis, which responds to the question posed in the title of this paper, is that children are citizens of the present and should be considered as an important group of the population. It was after the approval of the UN Convention on the Rights of the Child on 20 November 1989 that children's rights were recognised, particularly their right to participate in all matters affecting them. The study originated from the conviction that children are still not properly known, even though from the late 20th century their world has begun to receive greater social recognition. The main aim of the thesis was to identify children's participation in social life, analyse how they can influence new forms of participation, and relate this participation to the modern notion of citizenship. Ethnographic research methods were used to observe children in various settings where children were able to participate, as well as in-depth interviews with adults who specialise in children. This was also contrasted with bibliographic analysis. The paper exposes the concepts of childhood, participation and citizenship, offering a general view of some of the places where children can participate, which include the family, school and free time.
\end{abstract}

Key words: childhood, participation, citizenship.

\title{
I. ¿QUÉ ES LA INFANCIA?
}

Al plantearnos la consideración de los niños y niñas como ciudadanos, nos surge de entrada la pregunta de a quién nos estamos refiriendo. El punto de partida es el que establece la Convención Internacional de los Derechos del Niño que, en su artículo 1 refiere: "Para esta Convención se considera niño todo ser humano menor de 18 años, a no ser que en virtud de la ley que le sea aplicable, haya conseguido la mayoría de edad anteriormente a esta edad". Este planteamiento genera algunas incertidumbres, debido a que es difícil definir como infancia a aquellos a quienes les falta únicamente 2 ó 3 años para llegar a la mayoría de edad, pero, en realidad, uno de los factores que se consideran importantes cuando se piensa en los niños y niñas es precisamente el estatus jurídico de mayor de edad que confiere la plena capacidad de obrar. La realidad es que cada sociedad crea sus propios "estadios" de vida que pueden no existir en otras sociedades. Frecuentemente, si no se reconoce un determinado estadio del desarrollo, puede ser porque no existe para aquella sociedad. Por ejemplo, la adolescencia puede no existir en sociedades donde los niños y niñas tienen un alto nivel de emancipación de la familia, una identidad bien desarrollada y una capacidad cognitiva para operar de manera formal. En cambio, en otras culturas, -entre ellas, la española- es frecuente que, como destaca Ana Alabart (1998:56), los chicos y las chicas se queden en el hogar hasta mucho mayores debido a no encontrar un trabajo relativamente estable y al encarecimiento de los precios de las viviendas, sean estas de propiedad o en alquiler.

Si realmente la conducta de los niños está condicionada en parte por nuestras expectativas, su modificación puede conducir a diferentes conductas. Y si nuestras expectativas están influenciadas por las diversas teorías que, a la vez, reciben el impacto de cada cultura, podemos apercibirnos que estamos en una perspectiva muy amplia de construir la infancia: en nuestra relación con los niños y niñas no estamos relacionando sólo la "realidad" sino algo más complejo y dinámico. Al analizar cómo se ha construido socialmente la noción de infancia, no hay acuerdo entre diversos autores en determinar cuándo aparece la infancia como tal. Linda A Pollock (1993), en su estudio realizado sobre las relaciones entre padres e hijos de los siglos XVI al XX, muestra claramente que la infancia era ya contemplada antes del siglo XII, mientras que otros autores no la reconocieron hasta el siglo XX. Según el historiador Phillippe Ariès (1960-1985), la infancia no aparece hasta 
el siglo pasado. Antes, el niño era como un pequeño adulto que participaba plenamente en la vida social, sin tener ningún estatuto especial: trabajaba, vivía y formaba parte de forma plena en la sociedad.

Puede observarse que existen unas grandes oscilaciones en las interpretaciones históricas en relación con el reconocimiento de la infancia y las diversas posiciones de los autores (Robertson, 1994; De Mause, 1994; Freud, 1948; Van Gils, 1996). Es evidente que, como dice Pancera, "la búsqueda de la investigación histórica es estimulada en definitiva por el apremio que ciertas problemáticas nos hacen sentir. El descubrir otros modelos de relación compatibles con nuestra forma de civilización lleva a desvelar ocultaciones de aspectos de la realidad producidas por la ideología de las sociedades modernas y acerca de las cuales hasta ahora no habiamos reflexionado con detenimiento". Este historiador dirige la atención en el hecho que la investigación histórica también puede cumplir una función de tipo ideológico, legitimando la situación del momento o contradiciéndola y señala que "la producción del discurso histórico es, de hecho, enteramente del analista, es decir, del investigador, que habla de la Historia en nombre de su disciplina y de la Cultura" (Pancera, 1993:10).

En cualquier caso, es evidente que, en un determinado momento, la infancia ha ocupado un espacio claro y diferenciado y que es a partir de su descubrimiento que comenzaron a tomarse medidas para protegerlos del mundo de los adultos, iniciándose la creación de instituciones y leyes específicas para los niños y niñas. Se prohibió el trabajo infantil en muchos países y apareció la escolarización obligatoria. A partir de aquí, comenzó la creación de un mundo infantil sin riesgos ni responsabilidades donde los niños pudieran jugar y vivir seguros. Este hecho provocó la existencia de un mundo aparte, diferenciado, que, en cierta manera, ha alienado a los niños y niñas de la sociedad (Van Gils, 1996 (1):349). En la construcción social actual de la infancia, podemos afirmar que se tiende a verla como un sector de la población al que hemos de proteger o bien del que nos hemos de proteger. Micha de Winter (1997:10-15) presenta la evidencia de una tendencia a la problematización de la infancia en el siglo actual ${ }^{2}$. Toda la legislación específica dirigida a la infancia se ha caracterizado, hasta muy recientemente (como consecuencia de la aprobación de la Convención), en enfatizar la protección de la infancia por el hecho de que los niños y niñas son más débiles que los adultos y más influenciables a las posibles "malas compañías". No obstante, cuando se les ve como posibles delincuentes, se produce una gran contradicción: mientras se considera que los niños y niñas deben de protegerse constantemente y no tienen edad suficiente para participar en la vida social, cuando se trata de controlarlos porque sus actos pueden afectar a la seguridad ciudadana, se intenta equipararles al máximo a la edad adulta.

Esta visión proteccionista de la infancia ha podido dificultar la consideración de la participación de los niños y niñas en la vida social y llevar a considerar a la protección como opuesta a la participación. En realidad, son términos que se complementan, ya que la Convención de los Derechos del Niño, además de tratar de manera extensiva los necesarios aspectos de protección y provisión de servicios hacia la infancia, incorpora por primera vez, en un texto legal de máximo rango, una nueva visión de la infancia: la infancia que debe tener cubiertos los derechos de libertad de expresión y de participación en general, reconocidos a todos los ciudadanos, y es en este sentido que debe considerarse a los niños y niñas como tales.

2 El rechazo que generan los chicos/as como posibles delincuentes de los que el Estado debe proteger a los ciudadanos conllevó la dificultad que se aprobara la mayoría de edad penal a los 18 años y a la dificultad de aprobar la Ley Responsabilidad Penal del Menor, que va sufriendo múltiples modificaciones como respuesta a la reclamación que se penalicen de forma más dura las infracciones cometidas por menores. 


\section{II. ¿QUÉ SIGNIFICA LA PARTICIPACIÓN DE LA INFANCIA?}

La participación de la infancia debe enmarcarse en los movimientos de participación de toda la ciudadanía que se han producido en toda Europa a partir de los años 60 del pasado siglo. Este hecho está conectado al proceso de democratización a partir del cual diversos grupos reclaman más representatividad y ser escuchados en los procesos de toma de decisión de los asuntos que les afecten. En los años 80, en el Consejo de Europa, se trabajó el concepto de participación de los usuarios de servicios sociales ${ }^{3}$. En este sentido, un estudio realizado por Triseliotis, Borland, Hill \& Lambert (1995) en servicios de atención a la infancia, analizaba específicamente el nivel de participación de los adolescentes en la toma de decisiones, respecto a recibir atenciones específicas de los servicios sociales y judiciales y sobre su voluntad de ver o no a sus padres si se les había separado. Los resultados fueron que, de 116 , un $42 \%$ habían contribuido plenamente en la toma de decisiones, un $35 \%$ lo habían hecho de forma más limitada y un 16\% no habían sido consultados (el restante 7\% no había contestado). En relación con la estancia en centros residenciales, los jóvenes deseaban poder influir en las normas de funcionamiento, generalmente, solicitando que fueran menos estrictas, como, por ejemplo, en la hora de ir a dormir, la posibilidad de fumar y en la de ver a los amigos más frecuentemente ${ }^{4}$. Cuando se les preguntaba qué les gustaría ver en los adultos, muchos de ellos argumentaron que desearían que los adultos les trataran con mayor respeto y no como si fueran niños (Triseliotis et al, 1995:9). En relación con el sistema judicial, el estudio mostró que, en los juzgados juveniles de Inglaterra, los jóvenes no tenían ninguna expectativa de que se les preguntara cuáles eran sus puntos de vista y menos que se les tuviera en cuenta. En Escocia, donde el sistema judicial se supone que opera mayormente en la base de la participación buscando decisiones consensuadas y basadas en el bienestar de la infancia, la respuesta de los jóvenes sobre la oportunidad que tuvieron de participar no era demasiado positiva. Sólo un tercio consideró que habían podido dar su punto de vista y que éste se había tenido en consideración, pero los demás sentían que habían estado demasiado nerviosos para decir nada, que lo que habían dicho se había ignorado o que ni tan sólo habían tenido la oportunidad de hablar. Algunos decían que sólo hablaban los adultos y otros creían que el punto de vista de los padres había sido mucho más tenido en cuenta y que, frecuentemente, ellos eran considerados los culpables. Un chico decía claramente que: "siempre se preocupaban de lo que hacía y nunca pensaron en por qué lo hacia”" (Triseliotis et al, 1995:10).

A partir de este estudio, puede captarse qué esperan los chicos y chicas en base al concepto de participación y cómo ésta no cumple siempre las expectativas deseadas.

Diversos autores han tratado de manera extensiva el concepto de participación en la infancia (Hart, 1992; Rossini y Vulneau, 1992; Casas, 1994; Van Gils, 1996; Trilla y García, 2002). Todos ellos insisten en la importancia de que la participación sea real y no únicamente una simulación, que sería, como dice Hart (1992), aquellas situaciones en que aparentemente se da la voz a los niños y niñas, pero que, en realidad, no tienen ninguna posibilidad de ser realmente escuchados ni de dar sus propias opiniones. En este sentido, es de destacar la propia definición que dieron los propios niños y niñas en la II Audiencia Pública realizada en el Ayuntamiento de Barcelona el año 1997: "Participar es un derecho a manifestarse politicamente y socialmente sin que el hecho de ser menores de edad nos condicione. Es una actividad voluntaria que tomamos por propia iniciativa y con el deseo de expresar nuestras propias ideas. También sabemos que implica responsabilidad y compromiso. Participar es compartir, ayudar, ser solidario, comunicarnos, llegar a acuerdos

3 Los usuarios de los servicios sociales han sido especialmente considerados como "protegibles", pero, a la vez, excluidos del sistema del mismo modo que los niños y niñas.

4 Este tema sobre la mayor flexibilidad en las horas de ir a dormir fue también tratado de manera extensiva en las asambleas de los centros residenciales donde se realizó el trabajo de campo. 
comunes, trabajar conjuntamente con otros, conocer gente nueva, intercambiar opiniones y colaborar con tu entorno. Participar es tener opiniones, decir lo que piensas y ser escuchado. Es también tomar parte en la toma de decisiones". Los niños y niñas de Barcelona también destacaban que si no participaban más era porque tenían vergüenza, timidez, porque les parecía que los adultos no les harían caso y tampoco les daban suficiente espacio para hacerlo y también porque les faltaba información.

La participación tiene que ver con el ejercicio de la palabra y la posibilidad de los niños y niñas de actuar en todas aquellas áreas de su vida cotidiana que son de su interés. Cabe preguntarse qué es y qué no es de interés de la infancia. Toda la reciente legislación en materia de infancia se ha desarrollado sobre la base de la noción de "interés de la infancia" a la hora de tomar decisiones que interesen a los niños y niñas. Este concepto que justifica múltiples intervenciones que se hacen para la infancia y, como bien dice Encarna Roca (1994), es muy indeterminado, son generalmente los adultos los que tienden a darle contenido, cuando tendrían que ser los niños y niñas quienes pudieran manifestarse al respecto o al menos ser considerados. La Ley de la Generalitat de Catalunya 8/1995 sobre infancia ${ }^{5}$ impulsa que se tenga en cuenta a los niños y niñas y adolescentes en su interpretación. Los expertos entrevistados señalaron la dificultad de dar contenido a este concepto y la mayoría insistieron en que son los niños y niñas quienes tienen que dar su opinión, porque frecuentemente los propios adultos se sienten muy desconocedores de cuál puede ser o no su interés. Se relaciona el interés de la infancia precisamente con la participación, considerando que éste es uno de sus intereses principales ${ }^{6}$.

\section{EL CONCEPTO DE CIUDADANÍA VINCULADO A LA INFANCIA}

La ciudadanía es la condición relacionada con la pertenencia y participación en la organización política. Sus raíces se encuentran en la polis griega, donde este concepto se relacionaba con la situación jurídica de las personas consideradas miembros de la ciudad, condición indispensable para gozar de los derechos políticos de los que estaban privados los hombres que no tenían el status civitatis. En la antigua Roma, la ciudadanía plena - civis optimo iure-implicaba la posesión de unos derechos públicos que eran el ius sufragii (derecho a voto a las asambleas), el ius honorum (derecho a acceder a cargos públicos) y unos derechos privados, que eran el ius commercii (derechos de adquirir y transmitir propiedades y derecho de contratación en general) y el ius conubii (derecho a contraer matrimonio y constituir una familia con sus poderes inherentes que eran la tutela, la patria potestad y la capacidad de hacer testamento) (Iglesias, 1972: 137-141).

En la plasmación en el derecho positivo de las democracias modernas, la ciudadanía hace referencia a un conjunto de prácticas que otorgan la cualidad de componentes activos a los individuos de una comunidad de pertenencia. Sus beneficiarios son iguales respecto a los derechos y obligaciones que implica. Marshall (1950) consideraba que, aunque no existiera un principio universal que determinara plenamente cuáles son estos derechos y obligaciones, las sociedades donde la ciudadanía es una institución en desarrollo crean la imagen de una ciudadanía ideal que sirve para calcular el éxito y es objeto de las aspiraciones $^{7}$. En sus orígenes aristotélicos, la noción de ciudadanía estaba lejos de favorecer la inclusión de todos los seres humanos. Como destaca Dahrendorf, los primeros capítulos de

5 Ley 8/1995, de 27 de julio, de atención y protección a los niños y adolescentes y de modificación de la Ley 37/1991, de 30 de diciembre, sobre medidas de protección de los menores desamparados y de la adopción. (DOGC n. 2083 de 2.8.1995).

6 Pueden consultarse los resultados de la tesis en base al concepto del interés del menor de forma más específica en FERNÁNDEZ, J. “Los niños: ciudadanos participantes”, Revista de Treball Social, n. 179, diciembre 2006, pp. 52-56.

7 La monografía original de 1950 sobre ciudadanía de Th. Marshall fue publicada en castellano por Alianza Editorial, en 1998, conjuntamente con un capítulo titulado: "Ciudadanía y clase social, cuarenta años después" de Tom Bottomore. 
la Política abundan en afirmaciones que actualmente consideraríamos totalmente inaceptables. Para Aristóteles, los esclavos no eran ciudadanos porque no eran seres libres, como tampoco lo eran las mujeres, porque se las consideraba inferiores a los hombres "por naturaleza". Los niños, aunque fueran hijos de hombres libres, sólo serían ciudadanos cuando se hicieran mayores al llegar a la mayoría de edad (Dahrendorf, 1990:50).

El concepto de ciudadanía actual debe verse desde una perspectiva dinámica y más allá de las demarcaciones de un Estado. Es preciso ampliar el concepto de ciudadanía en base a diversos factores (Borja, 1998): a) por un lado, deben regularse de manera positiva unos verdaderos derechos "universales"; b) la redefinición "feminista" del concepto de ciudadanía en base a la cual, a pesar de los esfuerzos invertidos en la Declaración Universal de los Derechos Humanos, aún existen importantes factores de desigualdad jurídica, social, política y cultural entre los géneros y otros grupos sociales (en esta desigualdad también podemos situar a la infancia); y c) la necesidad de expandir la ciudadanía en el marco de los Estados y las organizaciones supra-estatales a aquéllos que por no ser nacionales de aquel Estado no gozan de plena ciudadanía. Aparece la necesidad de separar el concepto de ciudadanía del de nacionalidad. Respecto a la infancia, es preciso separar el concepto de ciudadanía del de mayoría de edad.

Es evidente que, en las sociedades modernas actuales, que tienen un alto nivel de complejidad y más necesidades a cubrir, el lenguaje de los derechos debe complementarse con el de responsabilidades. Experimentamos una nueva visión de la ciudadanía en la que derechos y deberes son complementarios y donde se plantea la obligación cívica de implicarse en los asuntos de la comunidad. La ciudadanía desea hacer llegar su voz en todos aquellos asuntos que les afectan y/o interesan (Inglehart, 1991). Esta nueva situación requiere una adaptación de las estructuras representativas para hacer posible una implicación más directa en la discusión de los problemas y en la participación en los mecanismos de toma de decisiones. La visión post-moderna de carácter individualista que caracteriza las sociedades globales conlleva una nueva posición de la ciudadanía que requiere un mayor protagonismo y mayor capacidad de intervención. Es en este sentido que la noción de ciudadanía está plenamente inter-conectada con la de participación.

La visión de la ciudadanía es, en este sentido, una figura central de las sociedades modernas que se completa con el valor de la pluralidad que implica respetar la diversidad de opiniones y comportamientos, estar dispuesto a asumir responsabilidades tanto a nivel grupal como en las estructuras organizativas y políticas y poder defender los derechos civiles, en caso de que en algún momento se cuestionaran. Es una ciudadanía relacionada con el concepto de democracia deliberativa que precisa ciudadanos y ciudadanas capaces de participar en las discusiones, con las mismas oportunidades, con los conocimientos suficientes como para tomar posiciones bien informadas y con disposición a asumir la tarea que les corresponda en cada momento ${ }^{8}$.

En relación a los niños y niñas como ciudadanos, como ya se ha visto, la Convención de los Derechos del Niño significa una base importante para su reconocimiento, ya que, fundamentándose en los artículos 12 al 17, pueden reclamar estar implicados de manera activa en los temas que les interesen a ellos directamente así como a su entorno social. La participación de la infancia es un medio imprescindible y necesario para que puedan ejercer esta ciudadanía. Esta participación tiene una doble vertiente: de una parte, contribuye a empoderar ${ }^{9}$ a los niños y niñas y hacerles capaces de articular y ser conscientes de cuáles son sus necesidades, de otra parte, es una forma de contribuir en la construcción y reconstrucción de esta nueva visión de la ciudadanía en la que han de participar tanto la infancia

8 Una interesante exposición sobre la democracia deliberativa y la ciudadanía es la realizada por Adela Cortina en: "Democracia deliberativa", EL PAIS, martes, 24 de agosto de 2004.

9 Me permito utilizar este sustantivo que traduzco del empowerment inglés. 
como los adultos. Moro defiende claramente la consideración del niño como "pienamente citadino" porque está inmerso en una red de relaciones sociales que estructuran su personalidad individual y social, tiene derechos y deberes, no únicamente en las relaciones con su familia sino también en las relaciones con toda la sociedad; tiene ya una identidad, y tiene una autonomía, aunque sea limitada, que debe desarrollar progresivamente. Se le considera un ciudadano precisamente ligado a este nuevo concepto de ciudadanía que implica no únicamente la pertenencia a una comunidad política y el conjunto de derechos y deberes derivados de ésta, sino también una relación particularmente intensa entre individuo y comunidad, entendida ésta desde una doble perspectiva: la participación plena y responsable de la ciudadanía en la vida social exigiendo sus derechos, pero igualmente cumpliendo sus deberes. El autor explica que el tema de la ciudadanía de la infancia no puede reducirse al ejercicio de algunos derechos políticos, sino que también supone considerar a estos niños y niñas como titulares de unos derechos que deben de poder ejercerse y que les hacen partícipes del desarrollo colectivo (Moro, 1996:239-243).

No obstante, la ciudadanía no se decreta, se conquista (Sancho-Andreo, 1997). Se pueden visualizar con claridad algunos factores que dificultan la creación de espacios de participación de la infancia:

- El hecho de que la participación en general es difícil para todo el mundo.

- El hecho de pertenecer a una minoría étnica, social o cultural.

- El hecho de ser niño en unas estructuras donde los adultos ocupan los lugares de toma de decisiones.

- El funcionamiento excesivamente rígido de las estructuras públicas.

- La visión que tienen muchos adultos de la infancia, relacionándola con la falta de madurez y de experiencia.

- El temor a que sean manipulados a causa de la falta de formación o de información para comprender los mecanismos "clásicos" de participación o para dominar correctamente los mecanismos de toma de decisiones.

Existen dificultades para que la ciudadanía de la infancia pueda ser reconocida. Ennew (2000) destaca cómo la Convención de los Derechos del Niño puede ser objeto de interpretaciones diversas. Los legisladores que redactaron el texto finalmente aprobado tendían inicialmente a incorporar su derecho a opinar en aspectos más personales, como, por ejemplo, el matrimonio, escoger trabajo, tratamiento médico, educación y tiempo libre. La influencia de los países de Europa del Norte, y, curiosamente, también los Estados Unidos (que no han ratificado la Convención), fue la que originó que el redactado final de los artículos fuera el que se aprobó finalmente, reconociendo un amplio abanico de posibilidades participativas y de derechos a ejercer por la infancia. Respecto a la implementación de la convención, la mayor responsabilidad se centra en el Comité de los Derechos del Niño, que se creó para hacer su seguimiento. Según la opinión de este comité, el artículo 1.210 de la convención, que se centra en la visión de que los niños y niñas son sujetos activos de derechos, está relacionado con el convencimiento de que son actores sociales competentes. A la vez, plantean, basándose en los informes que les llegan de los diversos países miembros, cómo esta implementación es uno de los más importantes retos de la convención.

10 1. Los Estados miembros deben asegurar a los niños y niñas con capacidad de formarse su propio juicio el derecho a manifestar su opinión en todos los asuntos que les afecten. Las opiniones del niño deben ser tenidas en cuenta en base a su edad y madurez.

2. Con esta finalidad, el niño debe tener, especialmente, la oportunidad de ser escuchado en cualquier procedimiento judicial o administrativo que le afecte directamente, bien directamente o mediante un representante o de una institución adecuada, de acuerdo a las normas de procedimiento de la legislación nacional. 


\section{LOS ESPACIOS DE PARTICIPACIÓN DE LOS NIÑOS Y NIÑAS}

Del análisis del trabajo de campo realizado, surgieron diversos espacios donde puede ejercerse la participación de la infancia y, en consecuencia, posibilitar este ejercicio de la ciudadanía por parte de los niños y niñas. Se destacan por su importancia, en los resultados conseguidos: la familia, el ámbito educativo y los espacios de ocio y asociaciones.

\subsection{La familia}

La familia es un sistema de relaciones y es el nivel primario en el que el niño aprende a relacionarse. Las relaciones familiares se establecen mediante una combinación de factores que son biológicos, psicológicos, sociales y económicos. La familia, tal y como la entendemos hoy en día, es un concepto moderno que surge con el nacimiento de la subjetividad y la preponderancia conferida a la institución del matrimonio establecido en términos de libre elección (Ubieto, 1995; Brullet, 2002). Debe tenerse en cuenta que los cambios en la familia moderna están relacionados con una noción de ciudadanía políticamente más activa que requiere que estos cambios se orienten en la dirección de afirmar los derechos individuales y profundizar en la democracia familiar (Brullet, 2002). Partiendo de esta función reconocida a la familia de desarrollar la propia identidad personal e integrarse en los roles sociales, aceptando la responsabilidad social, pero, a su vez, ofreciendo apoyo a la creatividad e iniciativa individual, es evidente que la familia es un espacio fundamental a tener en cuenta para la participación de la infancia. Esta participación se destacó especialmente en la Recomendación de 1998 sobre la participación de los niños en la familia y la vida social. Dicha recomendación establece que es posible y deseable la participación en sus diversas formas y grados y a todas las edades. Esta participación se considera como una forma de diálogo que favorece las habilidades de negociación y la resolución pacífica de conflictos ${ }^{11}$. No obstante, también debe tenerse en cuenta que existe una diferencia en la posición de padres e hijos en la familia, ya que ésta se inicia por una asociación voluntaria entre un hombre y una mujer y, en cambio, los niños y niñas aparecen en la familia sin haber manifestado su decisión de entrar a formar parte de ésta (Purdy, 1992:134).

En las consideraciones que las NN.UU. realizaron el año 1994, como año internacional de la familia, se señaló especialmente que debía favorecerse un equilibrio de las responsabilidades familiares, promoviendo una mayor participación de los hombres en las tareas familiares. En ningún momento se manifestó que los niños y niñas tomaran más parte en estas tareas, lo que podría considerarse como una tendencia excesivamente protectora del papel de la infancia en la familia. La cooperación en las tareas domésticas podría considerarse un tipo de participación en la familia. En este sentido, un estudio realizado el año 1995, donde se analizaba cuál era el nivel de participación de los niños y niñas en la redefinición del trabajo doméstico en la nueva familia urbana española y realizado con familias con hijos menores de 13 años en el área metropolitana de Madrid, mostraba que la participación de los niños y niñas en las tareas propias, como sería ordenar la habitación y sus cosas, variaba según la edad y que su implicación en las tareas comunes no se había modificado en el nuevo modelo de familia derivado de la incorporación de la mujer a la vida laboral. Como conclusión, se consideraba que, en el proceso de cambio que va requiriendo una organización familiar con un liderazgo compartido entre el padre y la madre y una estructura de toma de decisiones más consensuada y mayor implicación del hombre en las tareas domésticas, no iba acompañado en el caso de los hijos, por una aplicación

11 Recomendación R (98) 8E del Comité de Ministros de los países miembros del Consejo de Europa de 18 de septiembre de 1998. 
más generalizada de modelos más simétricos de socialización de los roles familiares (Meil, $1997)^{12}$.

El estudio realizado en el marco de los valores y estilos de vida de los niños y niñas españoles en 1994 de las ciudades de Barcelona, Bilbao, Madrid, Málaga, Sevilla, Valencia y Zaragoza, se centró en las relaciones inter-generacionales en el seno de la familia y relacionaba la participación con la comunicación. Se consideraba que el hecho de que los niños y niñas se comuniquen con los otros miembros de la familia (padres y abuelos) es una forma de implicarse y tomar parte. Las autoras destacaban que la participación es básica en la comunicación familiar, ya que comporta la posibilidad de actuar. Los resultados mostraban que existen dificultades de tiempo para dedicar a los niños y niñas y también de éstos para comunicarse con sus padres y abuelos (Pérez y Cánovas, 1995).

Las personas que participaron en el estudio dieron una gran relevancia al espacio familiar como imprescindible para fomentar la participación y la ciudadanía de la infancia, pero, a su vez, destacaban su dificultad: "La familia es el espacio fundamental. Es donde el estilo, el proyecto y modelo de vida se aprende y empapa uno con este modelo (...) y después se traslada a todas partes (...), pero, si el niño pequeño, de muy pequeño, ya ve que este espacio está cubierto por la madre, o la hermana mayor, ya crece con la idea de que no hay nada hacer y que todo está decidido..." (P3). "La familia creo que es un núcleo básico, importante, social y un espacio donde la participación debe potenciarse... Pero la familia está ajustándose a muchos cambios (...), es difícil (...) y yo creo que es el núcleo central" (P5). Las vivencias de participación en la familia destacarían por el hecho de ser espacios de vida cotidiana, como se destacaba en el estudio de Pérez-Alonso y Cánovas (1995) sobre la comunicación inter-generacional: "Yo no soy de una familia demócrata, porque en aquella época no lo era nadie, pero tengo unos padres (...) que realmente escuchaban y, entonces, en los espacios de comidas, siempre hablábamos. Yo recuerdo que mi padre decía que venía a almorzar porque así se enteraba de lo que habiamos hecho en clase... Era una costumbre que todos explicaran lo que habian hecho en el colegio o qué había pasado aquel día si tenía ganas de explicarlo (...), del mismo modo que mi padre explicaba algo de su trabajo y mi madre algo de la familia... Esta comunicación era muy agradable y lo hemos ido haciendo a lo largo de la vida, que la gente explicara qué le estaba pasando..." (P1).

Otro elemento que debe tenerse en cuenta en relación con la participación en la familia es el derecho a la intimidad. Este derecho, especialmente reconocido en la convención, es imprescindible que sea preservado para que se dé un verdadero respeto entre los diversos miembros de la familia y se considere al niño y niña como sujeto. Este tema fue ampliamente debatido en los espacios observados en que participaban niños y niñas y también defendido por las personas entrevistadas: “ Lo que falta es diálogo (...), ver que todos los componentes de la familia somos seres humanos con el mismo derecho exactamente, y esto es lo que falta y, en consecuencia, tratas al niño como un ser humano y no le abres nunca un cajón (...), ni escuchas una conversación por pequeña que sea...” (P1). "En las familias, la participación es esta: implicarles en una dinámica en que a los chicos se les tenga en cuenta (...), respetar su forma de ser, su intimidad..." (P4).

\subsection{El ámbito educativo}

El ámbito educativo, la escuela, conjuntamente con otros espacios de acción socio-educativa, como pueden ser los centros de día y espacios residenciales, es otro de los espacios

12 Esta no implicación podría ser consecuencia de que aún no se produzca una participación real de la infancia en la familia y todavía se funcione con sistemas "proteccionistas" de evitar que los niños realicen estas tareas. Este estudio demostraba cómo en las clases sociales más bajas se daba una mayor participación, pero que no dependía del hecho de que la madre trabajara o no. 
considerados especialmente por la Recomendación (98) 8 sobre participación infantil. La convención regula el derecho de los niños a la educación y establece la obligatoriedad de implantar como mínimo la enseñanza primaria obligatoria y gratuita para todos ${ }^{13}$. La Constitución española, en su artículo 27.4, regula también la enseñanza obligatoria y, a su vez, establece que se ha de asegurar la participación efectiva de todos los sectores implicados en la enseñanza. Uno de estos sectores son los propios niños/alumnos. La escuela se convierte, en las sociedades modernas, en uno de los espacios básicos donde los niños y niñas tienen el derecho y obligación de asistir. Como afirmaba Ferrer i Guàrdia (1977:69) "los gobiernos se han preocupado siempre de dirigir la educación del pueblo y saben mejor que nadie que su poder está casi totalmente basado en la escuela”. Este interés en la educación, por parte de todos los gobiernos a lo largo de los tiempos, convierte a la escuela en uno de los espacios donde poder - o no- favorecer una auténtica participación de la infancia. En este sentido, es importante resaltar el valor de la escuela moderna y cómo ésta se fundamenta en la consideración de que "todo el valor de la educación reside en el respeto de la voluntad fisica, intelectual y moral del niño" (Ferrer i Guàrdia, 1977:74). Los principios participativos de Montessori, Dalton y Decroly iban en este mismo sentido.

Además del lugar donde se adquiere conocimiento, la escuela es donde se obtiene una considerable experiencia en la forma en que los niños se relacionan entre ellos y los adultos. Se aprenden valores cívicos como la democracia, el respeto, la responsabilidad, la pluralidad y la solidaridad. Si se instaura un serio diálogo con los niños y niñas y la escuela, sobre la propia escuela, por ejemplo, discutiendo sus propios programas y organización, los niños y niñas aprenden que su opinión es apreciada y que se respeta su contribución. Si este diálogo no existe y no se estimulan las propias iniciativas, sino que, incluso, se dificultan, la lección implícita que aprenden es: “iLa ciudadanía es fantástica, pero no debajo del techo de mi casa!” (De Winter, 1997: 98). La educación siempre se ha visto como un espejo de la sociedad. Los niños y niñas antes del siglo pasado no disponían de posibilidades educativas. La educación sólo era posible para los niños ricos. En la Edad Media, los niños de familias sencillas sólo podían acceder a la educación bajo la fórmula de aprendizaje de oficios (Pancera, 1993).

Van Gils propone, como elemento propiciador de la participación, que debe ser trabajado desde las escuelas, lo que él denomina PEC (participación, expresividad y creatividad). Estos tres elementos se hacen necesarios a partir de la interacción del niño/a con su entorno, lo que implica estimular "el descubrimiento del sentido". La realidad es que las escuelas se dirigen con frecuencia mucho más hacia el niño/a individualmente y no se preocupan de las interacciones que se producen entre la infancia. Se da más importancia al trabajo individual que a las acciones de relación. Para contrarrestar esta tendencia, Van Gils plantea que habrá muchas más interacciones si los niños y niñas tienen más oportunidades: a) de comunicarse entre ellos, b) si existe un ambiente estimulador y c) si tienen libertad de definir las situaciones (Van Gils, 1996).

Los espacios observados fueron los siguientes: consejos escolares, asambleas de escuela, y asambleas de centros residenciales. De la observación participativa en los consejos escolares, donde los alumnos deben participar por ley, pudo captarse una importante dificultad para incorporar plenamente a los alumnos hacia una verdadera participación. El nivel de intervenciones en las sesiones, por parte de los estudiantes, era bajo, pero, en gran parte, este hecho estaba directamente relacionado con el orden del día, que raramente incluía temas propuestos directamente por los propios alumnos. Uno de los chicos, miembro del CE de un instituto de enseñanza secundaria, comentaba que, teniendo en cuenta la diferencia numérica entre los diversos representantes ( 8 profesores más dirección, cuatro padres y cuatro alumnos más un representante del Ayuntamiento) podía deducirse clara- 
mente que quien dominaría sería el profesorado. Consideraban, además, que los temas ya se llevaban muy trabajados y decididos desde el claustro de profesores. A pesar de todo, la asistencia del alumnado era considerable y aseguraban su presencia siempre que estuvieran convocados a tiempo ${ }^{14}$. Las manifestaciones de los adultos entrevistados también ponían en evidencia que los CE son espacios difíciles y complejos. No obstante, una de estas personas explicaba una experiencia de éxito en el proceso de creación del CE en una escuela de primaria donde había trabajado y participado. La experiencia se había dado en un periodo inicial de la puesta en marcha del CE, según la aplicación de la LODE. Consideraba que la pérdida de participación de los alumnos más pequeños y la no-obligatoriedad de la participación de los alumnos en las escuelas de primaria, según la LOGSE, había representado un retroceso: "Hicimos toda una planificación de trabajo bastante exhaustiva con los niños sobre cómo se tenía que participar, cómo se tenía que dinamizar el grupo y fue muy interesante. El grupo, después de estar unos días trabajando sobre cómo participar y el papel de la escuela (...) habia un consejo escolar donde ellos podian participar..." (P6). Esta experta del mundo educativo mostraba cómo, a partir de estar informados y participar en las elecciones, se constituían grupos-clase que convocaban sus propias asambleas para preparar los temas a aportar al CE. También se destacaba, como dato importante, que se les avisaba con tiempo suficiente para poder preparar los temas y pedir que se incorporaran en el orden del día.

A pesar de las dificultades, puede considerarse que los CE podrían favorecer - una vez salvados todos los obstáculos- una inclusión de los niños y las niñas en las organizaciones escolares, haciendo posible que florezca una cultura que haga más visible a la infancia. Respecto a las asambleas de escuela, éstas permitían especialmente el aprendizaje de la participación, el diálogo y el debate. No implicaban ninguna toma de decisión, pero sí que representaban la posibilidad de dialogar e interrelacionarse entre los compañeros y compañeras y tomar posiciones y opiniones sobre temas de importancia social. Probablemente, el factor de ser espacios didácticos favorecía que el profesor tuviera un rol menos directivo; en parte, porque era una persona realmente convencida de la necesidad de fomentar que los niños y niñas pudieran intervenir y aportar sus puntos de vista en los temas de su interés ${ }^{15}$. Estos espacios se configuraban con unas normas dirigidas a aprender el funcionamiento de los foros "clásicos" de debate que, incluso, se relacionaban con un Parlamento, con sus virtudes y defectos, como cuando, en ocasiones, el profesor les recordaba que, a veces, estaban aburridos. Las apreciaciones críticas que se daban en cuanto al sistema más rígido de funcionamiento llevan consigo un cuestionamiento de los sistemas clásicos de participación de nuestras sociedades democráticas, decantándose hacia sistemas de democracias participativas, los nuevos movimientos sociales y un nuevo concepto de ciudadanía. Pueden ser considerados como espacios que correspondían a unos diseños curriculares en relación a la vida cotidiana de los niños y su comunidad (Hart, 1997) y que favorecían la comunicación entre los alumnos (Van Gils, 1996).

Las asambleas de centro eran unas asambleas que se realizaban semanalmente para tratar temas de la vida cotidiana, en las que participaban los niños y niñas residentes y los educadores y educadoras. Dos de los educadores que participaban en dichas asambleas las definían así: "es un espacio compartido por menores y educadores". Es un espacio de decisión, de relación, de aprendizaje y de participación. Los temas pueden ser propuestos por

14 En algunas ocasiones, sobre todo al inicio, falló la convocatoria a los estudiantes, a quienes se avisaba el mismo día en que se realizaba la reunión.

15 El posicionamiento de los adultos frente a la participación de la infancia es especialmente trascendente para favorecerla, como se demuestra en todos los espacios estudiados. Para más detalle sobre esta posición de los adultos, ver: FERNÁNDEZ, J. "El dret dels infants a participar. Les condicions i condicionants d'aquesta participació". Revista Temps d'Educació. ICE Universitat de Barcelona. De inminente publicación en el número 37 correspondiente al mes de septiembre de 2009. 
todos sobre cuestiones relativas a la elaboración, la convivencia, la normativa, la gestión o la información (por ejemplo, decidir cómo recibir a un niño nuevo y quién será el encargado/a de presentarle la institución). Las decisiones, una vez tomadas, son vinculantes. El papel del educador es el de guía, facilitador y canalizador y tiene autoridad educativa. En la misma asamblea, se designa quién será el moderador y quién tomará acta (Gordaliza y Planas, 1996:80-81). Estas asambleas posibilitaban realmente la participación de los niños y niñas, pero existía aún una preponderancia de la intervención de los adultos y, en ocasiones, era difícil que se respetaran los acuerdos tomados. Los niños y niñas esperaban cada día con muchas ganas asistir a la asamblea. Al final de cada una, se hacía una valoración, mostrando todos una gran capacidad de valorar cómo había ido. Por consiguiente, a pesar de sus dificultades, era evidente que eran de interés de los niños y niñas, que reconocían su valor. En algunas ocasiones, se producían expulsiones si no se comportaban y, cuando esto se producía, todos manifestaban su interés en continuar y se quejaban de ser alejados del espacio. Los debates y opiniones les ofrecían la oportunidad de probar sus capacidades, en ocasiones, manifestar sus preocupaciones y hacer propuestas en relación especialmente a temas de la vida cotidiana. En lenguaje pedagógico, se diría que "aprendían de la experiencia", pero la realidad es que tenían la oportunidad de estar en un espacio participativo de vida cotidiana.

Estas asambleas tenían una cierta similitud a las reuniones-debate que hacia Korczak en la casa de huérfanos en el ghetto judío de Varsovia ${ }^{16}$. Él ya destacaba la importancia de estos debates para movilizar la conciencia colectiva y ayudar a resolver ciertos problemas particularmente espinosos o dolorosos. Insistía también en que se tenía que ir con cuidado en que no fueran "parodias de debate". Defendía que debía permitirse que los niños y niñas se expresaran con toda franqueza y que lo que dijeran en la reunión no fuera motivo de posteriores sanciones. A la vez, insistía en la importancia de que fuera una participación voluntaria y que nunca fuera una obligación (Korczak, 1999:320-321).

\section{3. Los espacios de ocio y las asociaciones}

La Recomendación (98) 8 del CE plantea, como uno de los espacios espacialmente idóneos para favorecer la participación de la infancia, a las actividades extra-escolares y, especialmente, todas las actividades de tiempo libre. También se reclama que se promuevan las asociaciones infantiles, facilitando el marco legal y los recursos necesarios para constituirlas. Les actividades de ocio de los niños y niñas están muy relacionadas con el juego, especialmente en las primeras edades. Tonucci afirma que "el juego del niño, consiste en "perder el tiempo», en perderse en el tiempo y encontrarse con el mundo mediante una relación excitante, llena de misterio, de riesgo y de aventura. El juego libre y espontáneo del niño tiene mucho que ver con las experiencias más sublimes y extraordinarias del adulto, como es la investigación, la exploración, la creación artística o la contemplación mística; equivale a las experiencias de las personas cuando se encuentran ante la complejidad, que tienen la posibilidad de dejarse transportar por el poderoso motor del placer". Tonucci las confronta con las propuestas educativas, señalando que, estas últimas, están a un nivel mucho más bajo, debido a que están provocadas y dirigidas por el adulto (1997:40). Hart (1997) destaca que para diseñar programas para la infancia, es importante saber cómo se reúnen de forma espontánea en grupos para intercambiar intereses, cómo se forman las amistades y cómo desarrollan un sentimiento de pertenencia. El autor alerta que se podría pensar que el mejor lugar para que se consideren dignamente los principios

16 Janusz Korczak (1879-1942), médico polaco, creó en Varsovia dos orfelinatos mixtos que funcionaban como "repúblicas infantiles", con un tribunal y un parlamento donde se aplicaban a todos las mismas reglas, incluidos los educadores y el director. No quiso dejar a los niños judíos que tenía a su cargo y murió con ellos en el campo de concentración de Treblinka. 
democráticos, por parte de la infancia, podría ser las organizaciones de tiempo libre fuera de la escuela. Considera que, desgraciadamente, la democratización de estos espacios es más la excepción que no la regla. Se destaca que, curiosamente, las mejores experiencias se encuentran en instituciones que se han establecido en los países industrializados para niños que han tenido problemas con la ley y que, en los países del Sur, muchas de las organizaciones que trabajan con los niños de la calle también tienen estructuras más democráticas (Hart 1997:63-64). Respecto al juego, Hart nos dice que, cuando los niños y niñas juegan, si se les observa detenidamente, todo lo que hacen, en un espacio donde cuentan con bastantes materiales, favorece una experimentación para la participación: aprenden las propiedades de los materiales, desarrollan habilidades físicas, exploran la utilización de las herramientas y a cooperar socialmente. A la vez, resalta que existen pocas oportunidades para el juego al aire libre a causa de una combinación de fuerzas: el miedo por la inseguridad de los espacios abiertos, las pautas de trabajo de los padres y la presión del aprendizaje académico. Hart considera que los niños y niñas que han tenido la oportunidad de participar en espacios cooperativos, tendrán más capacidad de trabajar conjuntamente en proyectos comunitarios con otros niños y adultos, que los niños y niñas que utilizan su tiempo en clases tradicionales en la escuela con grandes limitaciones de utilizar su tiempo libre de una forma no regulada (Hart, 1992:23-25).

La participación en movimientos infantiles y juveniles de tiempo libre es también uno de los espacios de especial consideración. La relación entre la participación y la responsabilización de los niños y niñas en estos movimientos y organizaciones fue uno de los argumentos importantes defendidos por Freire (1974) para favorecer un verdadero "empoderamiento" de la infancia. También llamaba la atención que, a su vez, este poder no tuviera como efecto que los oprimidos pasaran a ser opresores ${ }^{17}$.

En Cataluña, la incidencia de estos movimientos de tiempo libre es importante. De hecho, antes de llegar la democracia, habían sido los espacios más importantes donde los niños y niñas tenían la oportunidad de convivir y participar. Joan Costa (1997), en su tesis doctoral sobre estos movimientos en tiempos del Franquismo, demostró que habían sido escuelas de democracia y cómo muchos de los políticos catalanes de la primera época habían sido participantes activos de estos movimientos ligados preferentemente al Escultismo y la Acción Católica. Mar Galceran (2000), en su tesis doctoral sobre la participación de la infancia en espacios de ocio, demostraba que estos espacios organizados ofrecían buenas oportunidades de participación y cómo favorecían la construcción de un sentimiento de pertenencia a una colectividad. El estudio realizado sobre la infancia y las familias en el siglo XXI demuestra que más de 90.000 chicos y chicas catalanes se benefician de la acción de estos movimientos y las entidades que los organizan (Trilla y García, 2002: 46).

Estos espacios de tiempo libre, tanto los organizados como los que no lo son, deben ser especialmente considerados al analizar las oportunidades que tienen los niños y niñas de ejercer la ciudadanía. Se podría decir que es uno de los ámbitos por excelencia, puesto que el juego es muy importante, especialmente, cuando se produce de manera libre y espontánea. Los adultos entrevistados dieron mucha importancia a estas experiencias y asociaron inmediatamente la relación entre participación y sus propias experiencias infantiles con los espacios que disfrutaron de tiempo libre, que era cuando tenían la posibilidad de funcionar de manera autónoma y según quisieran ${ }^{18}$. Una de las entrevistadas relacionó sus experiencias de participación con todas las actividades que hacía libremente en verano y en la calle, la organización de verbenas y realización de hogueras que les permitieron

17 Esta preocupación se manifiesta con frecuencia por parte de los adultos, en relación a los niños y niñas, cuando se les visualiza como "pequeños tiranos".

18 Con la finalidad de captar el nivel de recuerdos que tenían los propios adultos sobre su propia infancia y, específicamente, sobre su noción de participación infantil como una vivencia propia, se les preguntaba qué experiencias de participación consideraban que habían tenido en su infancia. 
organizarse a su aire: "Nos dejaban mucho a nuestro aire (...), no había ni un adulto que se metiera en medio, diciéndonos cómo teníamos que hacer la hoguera... iHabía concursos entre calles, organizados por los niños!..., y por los adolescentes (...). Los padres no se ponían a organizar los concursos de las hogueras (...), para nada... Yo recordaré toda la vida que se hacian una reuniones en la calle... ;Estábamos habituados a jugar en la calle!..., los adultos sólo nos daban la posibilidad de hacerlo" (P2). Otro de los expertos que había sido especialmente crítico con su familia, por no haberle dado posibilidad de expresarse, rescataba especialmente los espacios de tiempo libre como espacios de autoafirmación y experimentación: " Sólo tenia el espacio del verano, en que me dejaban en paz, porque se iban a la playa y, entonces, estábamos con los primos y teníamos árboles que eran casas y eran nuestros. Y subiamos cosas a los árboles porque, arriba, los padres no se atrevían a subir. Era el único espacio que realmente considerábamos nuestro... El resto de mi infancia, estoy hablando de los años 50 y 60, era la desolación en el terreno de la participación: ;nada!...”.

Estos espacios más libres y este interés por la experimentación se confronta con este exceso de proteccionismo, especialmente en la que se llama actualmente la sociedad del riesgo (Castel, 2004) y en la que se transmite un sentimiento de gran inseguridad. Este nuevo paradigma ha comportado que estos relatos en los que los niños y niñas de ayer jugaban en la calle sean en la actualidad prácticamente imposible y se hace más necesario favorecer espacios abiertos donde los niños y niñas puedan moverse con facilidad ${ }^{19}$.

\section{CONCLUSIONES}

La ciudadanía, vista como una figura de las sociedades modernas, relacionada con el valor de la pluralidad que significa respetar la diversidad de opinión y comportamiento de todas las personas, tengan la edad que tengan, estar dispuesto a asumir responsabilidades tanto a nivel individual como grupal, como en las estructuras organizativas y políticas, y la capacidad de defender los derechos civiles si se cuestionan, relacionada con la necesaria y progresiva participación de los niños y niñas en todos los ámbitos, nos sitúa, sin duda, en la consideración de los niños y niñas como ciudadanos de pleno derecho en nuestra sociedad del siglo XXI.

La participación de la infancia es un proceso iniciado claramente desde los diversos ámbitos: la familia, la escuela, la ciudad y las relaciones con la Administración, en general. No obstante, podemos señalar que los espacios organizados de participación de la infancia aún muestran dificultades en su correcto funcionamiento, aunque dichos espacios, aunque sea con reticencias y dificultades, van favoreciendo una mayor visibilidad de la infancia y van permitiendo que los niños y niñas puedan ir asumiendo un papel más activo.

\section{BIBLIOGRAFIA}

ALABART I VILÀ, A., "La ciudad y los jóvenes", Revista de Treball Social, Col·legi Oficial de Diplomats en Treball Social i Assistents Socials de Catalunya, Barcelona: 1998, n. 149, pp. 55-61.

ARIÈS, P., El niño y la vida familiar en el antiguo régimen. Ed. Taurus, Madrid: 1985 (traducido del original en francés del año 1960).

BORJA, J., Ciudad y ciudadanía. Dos notas. Institut de Ciències Polítiques i Socials, Barcelona, 2000.

19 Estos espacios abiertos en la calle son los que ya favoreció Tonucci en su experiencia llevada a cabo en la ciudad de Fano (Italia) en la que los niños reclamaban un mayor espacio en la ciudad. Posteriormente, estas experiencias van proliferando en diversos municipios también en España. 
BRULLET, C., "Les polítiques de conciliació de la vida professional i la vida familiar: el cas dels Serveis de Suport a les famílies", en LLUÍS FLAQUER (coord.), Informe sobre la situació de la família a Catalunya: un intent de diagnòstic. Secretaria de la Família, Departament de Benestar i Família, Generalitat de Catalunya, Barcelona: 2002, pp. 271-300.

CASAS AZNAR, F., "Participación de los niños en la sociedad", Revista de Treball Social, Col·legi Oficial de Diplomats en Treball Social i Assistents Socials de Catalunya, Barcelona: 1994, n. 134, pp. 115-117.

CASTEL, R., La inseguridad social: ¿qué es estar protegido?, Ed. Manantial, Buenos Aires, 2004.

COSTA I RIERA, J., Dels moviments de l'esglèsia a la militància política. Mediterrània, Barcelona, 1997.

DAHRENDORF, R., El conflicto social moderno. Ensayo sobre la politica de la libertad. Mondatori, Madrid: 1990 (original en inglés de 1988).

DE MAUSE, L., (comp.). Historia de la infancia. Alianza Editorial, Madrid: 1982, 2a reimpresión en 1994.

DE WINTER, M., Children as fellow citizens, participation and commitment. Radclife Medical Press, Oxon, New York, 1997.

ENNEW, J., "How can we define citizenship in childhood?", Harvard Center for Population and Development Studies, Working Paper Series, Volume 10, n 12, octubre 2000.

FERNÁNDEZ I BARRERA, J., Els infants com a ciutadans, tesis doctoral defendida en la Universidad de Barcelona el año 2005, consultable en http://www.tesisenxarxa.net/TDX-0113109-110853/

FERNÁNDEZ I BARRERA, J., "Los niños: ciudadanos participantes” Revista de Treball Social, Col·legi Oficial de Diplomats en Treball Social i Assistents Socials de Catalunya, Barcelona: 2006, pp. 47- 56.

FERNÁNDEZ I BARRERA, J., "El dret dels infants a participar”, Revista Temps d'Educació, Universitat de Barcelona. ICE. Pendiente de publicación en el n. 37, Barcelona: 2009.

FERRER I GUARDIA, F., La Escuela Moderna. Editorial Zero, S.A., Bilbao, 1977.

FREIRE, P., Concientización. Ed. Búsqueda, Buenos Aires, 1974.

FREUD, S., "Recuerdos infantiles y encubridores", en Psicopatología de la vida cotidiana, obras Completas, Tomo I, Editorial Biblioteca Nueva, Madrid: 1948, pp. 648-652.

GALCERAN, M., La participació en els centres de temps lliure. Tesis doctoral presentada en la Facultat de Pedagogia de la Universitat de Barcelona, 2000.

GORDALIZA, B.; PLANAS. P.V., "La participación en un centro residencial de acción educativa", Revista de Treball Social, Col-legi Oficial de Diplomats en Treball Social i Assistents Socials de Catalunya, Barcelona: 1996, n. 144, pp. $77-82$.

HART, R., Children's participation. From tokenism to citizenship. Inocenti Essays, núm. 4., UNICEF, Florencia: 1992.

HART, R., Children's participation: the theory and practice of involving young citizens in community development and environmental care. UNICEF, Earthscan Publications Limited, New York, 1997.

IGLESIAS, J., Derecho romano. Instituciones de Derecho privado, Ed. Ariel, Esplugues de Llobregat (Barcelona), 1972.

INGLEHART, R., El cambio cultural en las sociedades industriales avanzadas. Centro de Investigaciones Sociológicas Siglo XXI, Madrid, 1991.

KORCZAK, J., Com estimar l'infant. Ed. Eumo. Universitat de Vic. Vic, 1999.

MARSHALL, T.H.; BOTTOMORE, T., Ciudadanía y clase social. Alianza Editorial, Madrid, 1998.

MEIL LANDWERLIN, G., "El papel de los niños en la redefinición del trabajo doméstico en la nueva familia urbana española", Revista Internacional de Sociología (RIS), Instituto de Estudios Sociales Avanzados, Madrid, n. 16, pp.39-56. 
MORO, A.C., "Il bambino come cittadino", en M.Á. VERDUGO; V. SOLER-SALA (eds), La convención de los Derechos del niño hacia el siglo XXI. Ediciones Universidad de Salamanca, Salamanca:1996, pp. 239-243.

PANCERA, C., Estudios de historia de la infancia. Promociones y Publicaciones Universitarias S.A. (PPU), Barcelona, 1993.

PÉREZ ALONSO-GETA, P.M.; CÁNOVAS LEONHARDT, P., "Relaciones familiares y valores: análisis intergeneracional", Infancia y sociedad, Ministerio de Asuntos Sociales. Dirección General del Menor y la Familia, Madrid, 1995, n. 29, pp. 117-145.

POLOCK, L., Los niños olvidados. Relaciones entre padres e hijos de 1500 a 1900. Fondo de Cultura Econòmica, Mèxico, 1993.

PURDY, L.M., In their best interest? The case against equal rights for children. Cornell University Press, Ithaca, New York, 1992.

ROBERTSON, P., "El hogar como nido: La infancia de la clase media en la Europa del siglo XIX”, en DE MAUSE, L. (comp.), Historia de la infancia. Alianza Editorial, Madrid, 1982, 2ª reimpresión 1994, pp. 444-471.

ROCA I TRIAS, E., “Contestació al discurs d'ingrès de la Dra. Alegria Borràs a l'Acadèmia de Jurisprudència i Legislació de Catalunya", Revista Jurídica de Catalunya, Il-lustre Col-legi d'Advocats de Barcelona. Academia de Jurisprudència i legislació de Catalunya, Barcelona, 1994, n. 4., pp. 101-122.

ROSSINI, N.; VULBEAU, A., Les conseils municipaux d'enfants et de jeunes, a la recherche d'un dispositif de participation. ANACEJ, Paris, 1992.

SANCHO ANDREO, R., "L'enfant acteur dans la cité et citoyen du monde". Conferencia presentada en el Primer encuentro de ayuntamientos por la Carta Municipal de los Derechos de Niños y Niñas, Málaga, 9-10 octubre, 1997.

TONUCCI, F., La ciutat dels infants. Ed. Barcanova, Barcelona: 1997. Existe traducción en castellano: La ciudad de los niños: un modo nuevo de pensar la ciudad. Fundación Germán Sánchez Ruipérez, Madrid: 1997.

TRILLA, J.; GARCIA, I., "Infància, temps lliure i participació social", en Carme Gómez-Granell et al. (coord.), La infància i les famílies a l'inici del segle XXI, Volumen 3. CIIMU, www.ciimu.org, Barcelona, 2002, pp. 14-148.

TRISELIOTIS, J.; BORLAND, M; HILL, M.; LAMBERT, L. Teenagers and the Social Work Services. HMSO, Londres, 1995.

UBIETO PARDO, J.R., "La familia moderna: ¿crisis o diversidad?”, Rambla 12. Revista de l'Associació promotora del Treball Social, Barcelona, $\mathrm{n}^{\mathrm{o}}$ 7, junio 1995, pp. 21-28.

VAN GILS, J., "Social participation: beyond the effect of sweetness, with illustrations from the Project Snater", en E. VERHELLEN (ed.) Monitoring Children's Rights, Martinus Nijhoff Publishers, La Haya, Boston, Londres, 1996, pp. 347-356. 